POS PROCEEDINGS

\title{
Closing Talk: Chiral Dynamics 2009
}

\section{Barry R. Holstein*}

Department of Physics-LGRT,

University of Massachusetts,

Amherst, MA 01003

E-Mail: holstein@physics.umass.edu

I present a few aspects of chiral dynamics which are of personal interest.

Chiral Dynamics 2009

Bern, Switzerland

July 6-10, 2009

${ }^{*}$ Speaker. 


\section{Introduction}

As the last speaker at Chiral Dynamics 2009, it is my pleasure to thank the organizers for putting together such an interesting and successful meeting. Anyone who has ever organized a conference knows that when a meeting runs as smoothly as this one does, it is no accident but rather it is on account of meticulous planning.

However, while in many meetings the final talk is a conference summary, mine will not be. That is because

i) the organizers told me I didn't have to

ii) in answering the questions-should this conference be summarized? and should I be the one to do it?-I fall back on a theorem which says that whenever a question is asked in the title of a physics paper, the answer is always no.

iii) I was always intrigued by the Isgur technique. Nathan was a fine speaker and way asked to give many summary talks.He would always say something like "This was a great meeting there were so many interesting ideas presented that there is no way that I could present a reasonable summary, so I won't try. Rather I will discuss a few ideas of interest to me." This is the model I shall adopt.

In thinking about the evolution of the Chiral Dynamics meetings during the past fifteen years, it is interesting to compare the first meeting at MIT in 1994 with the present one at Bern. There are a number of important differences. At the MIT meeting, we were in the midst of a withering heat wave, with temperatures each day in the nineties, and the sessions (and meals!) all took place in the (air-conditioned) Kolcker room in the physics department. We all stayed, however, in the MIT dorms which were not air-conditioned.

In 2009, we have the much more seasonable 70 degree temperatures in Bern and we are staying in comfortable hotels. Sessions are held in spacious university lecture halls. Nevertheless some things haven't changed. That includes many of the people-myself, Aron Bernstein, Juerg Gasser, Heiri Leutwyler, Steve Weinberg, etc. Also, the same excitement about chiral physics which was prevalent at MIT is present here in Bern, and so in my talk I want to tell you about some of the ideas that excite me.

\section{2. $K \rightarrow 3 \pi$}

One subject which is of great interest to me is that of $K \rightarrow 3 \pi$ decays, which was also the subject of my 1969 thesis[1]. In this thesis I used what was called current algebra/PCAC methods which allowed one to relate the amplitude for $K \rightarrow 3 \pi$ decay in the limit as one of the pion's fourmomentum vanishes to an experimental $K \rightarrow 2 \pi$ amplitude[2]. One does this by parameterizing the $K \rightarrow 3 \pi$ via

$$
<\pi^{+} \pi^{-} \pi^{0}\left|\mathscr{H}_{w}\right| K^{0}>=a+b s_{0}+c\left(s_{+}+s_{-}\right)+\ldots
$$

Now require

$$
\lim _{q_{\pi^{0}} \rightarrow 0}<\pi^{+} \pi^{-} \pi^{0}\left|\mathscr{H}_{w}\right| K^{0}>=\frac{-i}{F_{\pi}}<\pi^{+} \pi^{-}\left|\left[F_{5}^{3}, \mathscr{H}_{w}\right]\right| K^{0}>
$$


But since weak currents are left handed we have

$$
\left[F_{5}^{3}, \mathscr{H}_{w}\right]=\left[I_{3}, \mathscr{H}_{w}\right]
$$

so

$$
\lim _{q_{\pi^{0}} \rightarrow 0}<\pi^{+} \pi^{-} \pi^{0}\left|\mathscr{H}_{w}\right| K^{0}>=\frac{-i}{2 F_{\pi}}<\pi^{+} \pi^{-}\left|\mathscr{H}_{w}\right| K^{0}>
$$

and similarly for the limits as $q_{\pi^{+}}, q_{\pi^{-}} \rightarrow 0$. The results of this procedure were surprisingly good predictions for both amplitudes and slopes and all $K \rightarrow 3 \pi$ amplitudes[1].

Now fast forward to 1990. At this point Joachim Kambor, a student of Daniel Wyler, wrote his thesis on $K \rightarrow 3 \pi$ decays from the standpoint of chiral perturbation theory[3]. This was a substantial calculation since there are three terms at lowest $\mathbf{O}\left(q^{2}\right)$ order and forty-eight at $\mathscr{O}\left(q^{4}\right)$. Of course, these results must be consistent with the "old" current algebra/PCAC limits and using this constraint, I found a number of typos in the thesis-such methods can provide a useful check on chiral calculations[4].

It is interesting to ask why such a consistent one-loop calculation had not taken place before this time. Indeed Gasiorowicz and Geffen had written a comprehensive tree level chiral Lagrangian at the end of the 1960's[5] while Pagels and others had looked at the nonanalytic parts of the loop corrections during the 1970's[6], so in principle the Kambor analysis could have been done at that time. Why wasn't it? I think the reason is that at the time, especially because of the success of the Weinberg-Salam unification of weak and electromagnetic interactions, we were focused on the idea of producing interactions which were renormalizable. Only with Weinberg's 1979 paper[7], which emphasized the usefulness of nonrenormalizabile effective field theories, did this mindset change and this led to the development of chiral perturbation theory by Gasser and Leutwyler in 1984[8].

The current state of the art is evidenced in papers by Bijnens et al.[9], by Prades et al.[10], and by others which have extended the one loop corrections to include effects of isospin breaking and electromagnetic corrections. The results are detailed and very successful fits to very precise $K \rightarrow 3 \pi$ data including terms up to quadratic order.

\section{$2.1 \pi-\pi$ Scattering Lengths}

In the mean time there have also been interesting chiral developments in the area of pi-pi scattering lengths. In the isospin-symmetric limit, the $\pi-\pi$ scattering amplitude has the form

$$
T_{\alpha \beta ; \gamma \delta}(s, t, u)=\delta_{\alpha \beta} A(s, t, u)+\delta_{\alpha \gamma ; \beta \delta} A(t, u, s)+\delta_{\alpha \delta} \delta_{\beta \gamma} A(u, s, t)
$$

Then Weinberg's 1966 result from lowest order chiral symmetry is that[11]

$$
A(s, t, u)=\frac{s-m_{\pi}^{2}}{F_{\pi}^{2}}
$$

It is conventional to present these results in an isotopic spin basis wherein[12]

$$
\begin{aligned}
& T_{0}(s, t, u)=3 A(s, t, u)+A(t, u, s)+A(u, s, t) \\
& T_{1}(s, t, u)=A(t, u, s)-A(u, s, t) \\
& T_{2}(s, t, u)=A(t, u, s)+A(u, s, t)
\end{aligned}
$$


in terms of which we find the Weinberg scattering lengths

$$
a_{0}^{0}=\frac{7 m_{\pi}^{2}}{32 \pi F_{\pi}^{2}}, \quad a_{0}^{2}=-\frac{m_{\pi}^{2}}{16 \pi F_{\pi}^{2}}, \quad a_{1}^{1}=-\frac{m_{\pi}^{2}}{24 \pi F_{\pi}^{2}}
$$

In the case of $a_{0}^{0}$ this gives the result

$$
{ }^{L O} a_{0}^{0}=0.16, \quad{ }^{L O} a_{0}^{2}=-0.05
$$

In 1984 Gasser and Leutwyler determined the one loop chiral correction to be

$$
{ }^{N L O} a_{0}^{0}=0.20
$$

Then in 2001 Colangelo, Gasser, and Leutwyler presented the results of an all orders analysis using the Roy equations[13]

$$
{ }^{R o y} a_{0}^{0}=0.220 \pm 0.005 \quad{ }^{R o y} a_{0}^{2}=0.044 \pm 0.001
$$

In the meantime it had been pointed out by Stern and others that these values corresponded to the assumption that[14]

$$
\frac{<0|\bar{q} q| 0>}{F_{\pi}^{2}} \simeq 1 \mathrm{GeV}
$$

and that it was also possible that

$$
\frac{<0|\bar{q} q| 0>}{F_{\pi}^{2}}<<1 \mathrm{GeV}
$$

leading to the so-called "generalized chiral perturbation theory", whereby ${ }^{g}{ }^{p t} a_{0}^{0}=0.26$.

On the experimental side there have been two determinations of these S-wave scattering lengths. One is via use of the Fermi-Watson theorem to extract the scattering lengths from $K_{\ell 4}$ data, which yields[15]

$$
a_{0}^{0}=0,221 \pm 0.026
$$

More interesting and relevant to our discussion is the use of the cusp structure of final state interactions in $K \rightarrow 3 \pi$ to measure these scattering lengths. This phenomenon is perhaps better known in the analysis of neutral pion photoproduction in the threshold region[16], wherein there is interference between the direct amplitude for

$$
\gamma p \rightarrow \pi^{0} p
$$

and the rescattering diagram

$$
\gamma p \rightarrow \pi^{+} n \rightarrow \pi^{0} p
$$

The result for the $\mathrm{S}$-wave $\left(E_{)+}\right)$amplitude is

$$
E_{0+}\left(\gamma p \rightarrow \pi^{0} p ; s\right)=e^{i \delta_{0}(s)}\left[A(s)+i q_{+} \beta\right]
$$

where $q_{+}=\frac{1}{2} \sqrt{s-4 m_{\pi^{+}}^{2}}$ is the charged pion momentum and

$$
\beta \sim E_{0+}\left(\gamma p \rightarrow \pi^{+} n\right) a_{\pi^{+} n \rightarrow \pi^{0} p}
$$


Then above the $\pi^{+} n$ threshold $q_{+}$is real and

$$
\left|E_{0+}\left(\gamma p \rightarrow \pi^{0} p\right)\right|^{2} \propto|A(s)|^{2}-q_{+}^{2} \beta^{2}
$$

and is a smooth function of $s$. On the other hand in the region

$$
\left(m_{p}+m_{\pi^{0}}\right)^{2}<s<\left(m_{n}+m_{\pi^{+}}\right)^{2}
$$

we have $q_{+}=i\left|q_{+}\right|$and

$$
\left|E_{0+}\left(\gamma p \rightarrow \pi^{0} p\right)\right|^{2} \propto|A(s)|^{2}-\beta^{2}\left|q_{+}\right|^{2}-2 A(s) \beta\left|q_{+}\right|
$$

The term linear in $\left|q_{+}\right|$produces the unitarity cusp and has been clearly seen in experiments at MAMI.

The same phenomenon occurs in the $K \rightarrow 3 \pi$ and $\eta \rightarrow 3 \pi$ systems. Specifically in the decay $K^{+} \rightarrow \pi^{+} \pi^{0} \pi^{0}$, there is an interference between the direct amplitude and the rescattering diagram involving $K^{+} \rightarrow \pi^{+} \pi^{+} \pi^{-} \rightarrow \pi^{+} \pi^{0} \pi^{0}$. This was seen experimentally at Frascati and was interpreted by Cabibbo[17]. Later analysis by Cabibbo and Isadori[18] and by a Bern collaboration has led to a measurement of both $a_{0}$ and $a_{2}$ [19]. Preliminary results from NA48/2 are[20]

$$
a_{0}=0.261 \pm 0.006 \pm 0.003 \pm 0.013 \quad \text { and } \quad a_{2}=-0.037 \pm 0.009 \pm 0.013
$$

and are in good agreement with the predictions of conventional chiral perturbation theory.

\section{Electromagnetic Polarizabilities}

A longtime interest of mine has been that of electromagnetic polarizabilities. The simplest of these are the electric (magnetic) polarizability $\alpha_{E}\left(\beta_{M}\right)$ which is the constant of proportionality between the induced electric (magnetic) dipole moment and an applied electric (magnetic) field[24]. In the case of the electric field we have

$$
\vec{p}=4 \pi \alpha_{E} \vec{E}
$$

which corresponds to an energy density

$$
u_{E}=-2 \pi \alpha_{E} \vec{E}^{2}
$$

while in the case of a magnetic field we have

$$
\vec{m}=4 \pi \beta_{M} \vec{H}
$$

which corresponds to an energy density

$$
u_{M}=-2 \pi \beta_{M} \vec{H}^{2}
$$

One can measure the polarizability of an elementary system by Compton scattering. That is, for a particle of charge $Q$, the Hamiltonian which describes the interaction with an external electromagnetic field is

$$
H=\frac{(\vec{p}-Q \vec{A})^{2}}{2 m}-\frac{1}{2} 4 \pi \alpha_{E} \vec{E}^{2}-\frac{1}{2} 4 \pi \beta_{M} \vec{H}^{2}+\ldots
$$


which leads to a Compton scattering amplitude

$$
T=\hat{\varepsilon} \cdot \hat{\varepsilon}^{\prime}\left(-\frac{Q^{2}}{m}+\omega \omega^{\prime} 4 \pi \alpha_{E}\right)+\hat{\varepsilon} \times \vec{k} \cdot \hat{\varepsilon}^{\prime} \times \vec{k}^{\prime} 4 \pi \beta_{M}+\ldots
$$

This leads to a cross section

$$
\frac{d \sigma}{d \Omega}=\frac{\alpha^{2}}{m^{2}}\left(\frac{\omega^{\prime}}{\omega}\right)^{2}\left[\frac{1}{2}\left(1+\cos ^{2} \theta\right)-\frac{m \omega \omega^{\prime}}{\alpha}\left(\frac{\alpha_{E}+\beta_{M}}{2}(1+\cos \theta)^{2}+\frac{\alpha_{E}-\beta_{M}}{2}(1-\cos \theta)^{2}\right)\right]
$$

so that by measuring the differential cross section one can extract values of the electric and magnetic polarizabilities. In the case of the proton, a series of measurements at MAMI, Saskatoon, and Illinois have yielded the values[21]

$$
\alpha_{E}^{p}=(12.0 \pm 0.6) \times 10^{-4} \mathrm{fm}^{3} \quad \text { and } \quad \beta_{M}=(1.9 \mp 0.6) \times 10^{-4} \mathrm{fm}^{3}
$$

These are fundamental properties of the proton we have learned a number of things from such measurements:

i) the electric polarizability provides a measure of the "stiffness" of a system. In the case of the hydrogen atom, there exists an exact result[22]

$$
\alpha_{E}^{H}=\frac{9}{2} a_{B}^{3}=\frac{27}{8 \pi} \text { Vol. }
$$

where $a_{B}$ is the Bohr radius and Vol. $=\frac{4}{3} \pi a_{B}^{3}$ is the volume. In the case of the proton we have

$$
\alpha_{E}^{p} \sim 3 \times 10^{-4} \text { Vol. }
$$

which says that the proton is a much stiffer system than a hydrogen atom. This can be understood in a handwaving fashion by noting that

$$
\frac{\alpha_{E}^{p} / \text { Vol. }}{\alpha_{E}^{H} / \text { Vol. }} \sim \frac{E_{\text {bind }}^{H} / m}{E_{\text {bind }}^{p} / m} \sim \frac{\alpha_{\text {em }}^{2}}{\alpha_{\text {strong }}^{2}} \sim 10^{-4}
$$

ii) the $\Delta$ pole makes a strong paramegnetic contribution $\sim 10 \times 10^{-4} \mathrm{fm}^{3}$ [23] so there must be a strong diamagnetic contribution to cancel much of this

iii) this cancelation presumably comes from the pion cloud-indeed a simple valence quark model gives

$$
{ }^{\text {quark }} \alpha_{E}^{p}=2 \alpha m_{p}<r_{b}^{2}>^{2}>>{ }^{\exp } \alpha_{E}^{p}
$$

The importance of the pion cloud suggest the use of chiral perturbation theory and Bernard, Kaiser, and Meissner have calculated[25]

$$
\begin{aligned}
& \alpha_{E}^{p}=\frac{\alpha g_{A}^{2}}{48 \pi^{2} F_{\pi}^{2} m_{\pi}}\left[\frac{5 \pi}{2 \mu}+18 \log \mu+\frac{33}{2}+\mathscr{O}(\mu)\right]=7.4 \times 10^{-4} \mathrm{fm}^{3} \\
& \beta_{M}^{p}=\frac{\alpha g_{A}^{2}}{48 \pi^{2} F_{\pi}^{2} m_{\pi}}\left[\frac{\pi}{4 \mu}+18 \log \mu+\frac{63}{2}+\mathscr{O}(\mu)\right]=-2.0 \times 10^{-4} \mathrm{fm}^{3}
\end{aligned}
$$


where $\mu=m_{\pi} / M_{p}$. If we retain only the leading piece here, which corresponds to $\mathscr{O}\left(q^{3}\right)$ heavy baryon chiral perturbation theory, we have

$$
\alpha_{E}^{p}=10 \beta_{M}^{p}=\frac{5 g_{A}^{2}}{96 \pi F_{\pi}^{2} m_{\pi}}=12.2 \times 10^{-4} \mathrm{fm}^{3}
$$

which is in spectacular agreement with experiment. However, this clearly accidental since an $\mathscr{O}\left(q^{4}\right)$ calculation yields[26]

$$
\alpha_{E}^{p}=(10.5 \pm 2.0) \times 10^{-4} \mathrm{fm}^{3} \quad \text { and } \quad \beta_{M}=(3.5 \pm 3.6) \times 10^{-4} \mathrm{fm}^{3}
$$

Of particular interest is the charged pion polarizability, where $\mathscr{O}\left(q^{4}\right)$ chiral perturbation theory predicts[27]

$$
\alpha_{E}^{\pi^{+}}+\beta_{M}^{\pi^{+}}=0 \quad \text { and } \quad \alpha_{E}^{\pi^{+}}-\beta_{M}^{\pi^{+}}=5.4 \times 10^{-4} \mathrm{fm}^{3}
$$

and a two loop calculation yields small corrections. On the experimental side the polarizabilities have been measured in three different ways:

i) a Primakoff effect measurement by Antipov et al. using the reaction $\pi^{+} Z \rightarrow \pi^{+} \gamma Z$ has given[28]

$$
\alpha_{E}^{\pi^{+}}=(6.8 \pm 1.4 \pm 1.2) \times 10^{-4} \mathrm{fm}^{3}
$$

ii) a MAMI experiment involving the reaction $\gamma N \rightarrow \gamma \pi^{+} N$ attempted to extrapolate to the pion pole and yielded[29]

$$
\alpha_{E}^{\pi^{+}}-\beta_{M}^{\pi^{+}}=(11.6 \pm 1.5 \pm 3.0 \pm 0.5) \times 10^{-4} \mathrm{fm}^{3}
$$

iii) a SLAC experiment utilizing the reaction $\gamma \gamma \rightarrow \pi^{+} \pi^{-}$has given[30]

$$
\alpha_{E}^{\pi^{+}}=(2.2 \pm 1.6) \times 10^{-4} \mathrm{fm}^{3}
$$

Obviously there is a problem here and the hope is that COMPASS will come to the rescue by redoing the Antipov experiment. We look forward to this important measurement.

The future of this field lies is the measurement of spin-dependent polarizabilities. These can be understood by realizing that if we consider the excitation and deexcitation of a system then the electric polarizability can be written as $\alpha_{E 1 E 1}$ while the magnetic polarizability is $\beta_{M 1 M 1}$. If we introduce spin, then four additional structure constants are involved and the effective Hamiltonian is[31]

$$
\begin{aligned}
H & =-\frac{1}{2} 4 \pi\left[\gamma_{E 1 E 1} \vec{\sigma} \cdot \vec{E} \times \dot{\vec{E}}+\gamma_{M 1 M 1} \vec{\sigma} \cdot \vec{H} \times \dot{\vec{H}}\right. \\
& \left.+2 \gamma_{E 1 M 2} \sigma_{i} E_{j} H_{i j}-2 \gamma_{M 1 E 2} \sigma_{i} H_{j} E_{i j}\right]
\end{aligned}
$$

where $E_{i j}=\frac{1}{2}\left(\nabla_{i} E_{j}+\nabla_{j} E_{i}\right)$. Heavy baryon predictions at $\mathscr{O}\left(q^{3}\right)$ are available

$$
\gamma_{E 1 E 1}^{p}=-5 \gamma_{M 1 M 1}^{p}=5 \gamma_{E 1 M 2}=5 \gamma_{M 1 E 2}=-\frac{1}{\pi m_{\pi}} \alpha_{E}^{p}
$$

However, each of these structure constants also has a large pion pole contribution

$$
\gamma_{E 1 E 1}^{p}=-\gamma_{M 1 M 1}^{p}=-\gamma_{E 1 M 2}=\gamma_{M 1 E 2}=\frac{2.4}{\pi m_{\pi} g_{A}} \alpha_{E}^{p}
$$

which must be subtracted off in order to reveal the dynamical quantities. A program in this regard is underway at Hi $\gamma_{\mathrm{s}}$ and at MAMI. We anxiously look forward to these measurements. 


\section{Conclusion}

These are only two of many interesting topics which have been covered at this meeting. On account of space limitations, we will end our discussion here.

\section{Acknowledgement}

This work was supported in part by the National Science Foundation under award PHY0553304.

\section{References}

[1] B.R. Holstein, Nonleptonic Kaon Decay, Carnegie-Mellon University PhD Thesis (1969).

[2] B.R. Holstein, Current Algebra Model of $\Delta I=3 / 2$ Effects in Nonleptonic Kaon Decay, Phys. Rev. 183, 1228 (1969).

[3] J. Kambor, J.H. Missimer, and D. Wyler, $K \rightarrow 2 \pi$ and $K \rightarrow 3 \pi$ Decays in Next-to-leading Order Chiral Perturbation Theory, Phys. Lett. B261, 496 (1991).

[4] J. Kambor, J.F. Donoghue, B.R. Holstein, J.H. Missimer, and D. Wyler, Chiral Symmetry Tests in Nonleptonic K Decay, Phys. Rev. Lett. 68, 1818 (1992).

[5] S. Gasiorowicz and D.A. Geffen, Effective Lagrangians and Field Algebras with Chiral Symmetry, Rev. Mod. Phys. 41, 531 (1969).

[6] See, e.g., P. Langacker and H. Pagels, Chiral Perturbation Theory, Phys. Rev. D8, 4595 (1973).

[7] S. Weinberg, Phenomenological Lagrangians, Physica A96, 97 (1979);

[8] J. Gasser and H. Leutwyler, Chiral Perturbation Theory to One Loop, Ann. Phys. (NY) 158, 142 (1984); Chiral Perturbation Theory: Expansions in the Mass of the Strange Quark, Nucl. Phys. B250, 465 (1985).

[9] H. Bijnens and F. Borg, Isospin Breaking in $K \rightarrow 3 \pi$ : 1. Strong Isospin Breaking, Nucl. Phys. B697, 319 (2004); 2. Radiative Corrections, Eur. Phys. J. C39, 347 (2005); 3. Bremsstrahlung and Fit to Experiment, Eur. Phys. J. C40, 383 (2005).

[10] E. Gamiz, J. Prades, and I Scimemi, Charged Kaon $K \rightarrow 3 \pi$ CP Violating Asymmetries at NLO in ChPT, JHEP 310, 42 (2003).

[11] S. Weinberg, Pion Scattering Lengths, Phys. Rev. Lett. 17, 616 (1966).

[12] J.F. Donoghue, E. Golowich, and B.R. Holstein, Dynamics of the Standard Model, Cambridge Univ. Press, New York (1992).

[13] G. Colangelo, J. Gasser, and H. Leutwyler, Pi Pi Scattering, Nucl. Phys. B603, 125 (2001).

[14] M. Knecht, B. Moussallam, J. Stern, and N.H. Fuchs, The Low Energy Pi Pi Amplitude to One and Two Loops, Nucl. Phys. B457, 513 (1995); Determination of Two Loop Pi Pi Scattering Amplitude Parameters, Nucl. Phys. B471, 445 (1996).

[15] G. Colangelo, J. Gasser, and H. Leutwyler, The Quark Condensate from $K_{e 4}$ Decays, Phys. Rev. Lett. 86, 5008 (2001).

[16] V. Bernard, N. Kaiser, and U.-G. Meissner, Threshold Pion Photoproduction in Chiral Perturbation Theory, Nucl. Phys. B383, 442 (1992). 
[17] N. Cabibbo, Determination of the $a_{0}-a_{2}$ Pion Scattering Length from $K^{+} \rightarrow \pi^{+} \pi^{0} \pi^{0}$ Decay, Phys. Rev. Lett. 93, 121801 (2004).

[18] N. Cabibbo and G. Isadori, Pion-pion Scattering and the $K \rightarrow 3 \pi$ Decay Amplitude, JHEP 503, 21 (2005).

[19] G. Colangelo, J. Gasser, B. Kubis, and A. Rusetsky, Cusps in $K \rightarrow 3 \pi$ Decays, Phys. Lett. B638, 187 (2006).

[20] S. Guidici, $\pi \pi$-Scattering Length Measurements from $K \rightarrow 3 \pi$ Decays, these proceedings (2009).

[21] M. Schumacher, Polarizability of the Nucleon and Compton Scattering, Prog. Part. Nucl. Phys. 56, 567 (2005).

[22] E. Merzbacher, Quantum Mechanics, Wiley, New York (1993).

[23] N.C. Mukhopadhyay, A.M. Nathan, and L. Zhang, Delta Contribution to the Paramagnetic Polarizability of the Proton, Phys. Rev. D47, 7 (1993).

[24] B.R. Holstein, Electromagnetic Polarizabilities of the Nucleon, Comm. Nucl. Part. Phys. 20, 301 (1992).

[25] V. Bernard, N. Kaiser, and U.-G. Meissner, Chiral Expansion of the Nucleon's Electromagnetic Polarizabilities, Phys. Rev. Lett. 67, 1515 (1991).

[26] V. Bernard, N. Kaiser, A. Schmidt, and U.-G. Meissner, Consistent Calculation of the Nucleon Electromagnetic Polarizabilities in Chiral Perturbation Theory Beyond Leading Order, Phys. Lett. B319, 269 (1993).

[27] B.R. Holstein, Pion Polarizability and Chiral Symmetry, Comm. Nucl. Part. Phys. 19, 221 (1990).

[28] Yu M. Antipov, et al., Measurement of the Pi-Meson Polarizability in Pion Compton Effect, Phys. Lett. B121, 445 (1983).

[29] J. Ahrens et al., Measurement of the Meson Polarizability via the $\gamma p \rightarrow \gamma \pi^{+} n$ Reaction, Eur. J. Phys. A23, 113 (2005).

[30] D. Babusci et al., Chiral Symmetry and Pion Polarizabilities, Phys. Lett. B277, 158 (1992).

[31] B.R. Holstein, D. Drechsel, B. Pasquini, and M. Vanderhaeghen, Higher Order Polarizabilities of the Proton, Phys. Rev. C61, 034316 (2000). 